\title{
Análisis prospectivo estratégico de la educación superior en Colombia
}

\section{Strategic prospective analysis of higher education in Colombia}

DOI: http://dx.doi.org/10.17981/cultedusoc.11.1.2020.13

Recibido: 19/11/2019. Aceptado: 19/02/2020. Publicado: 23/03/2020

\section{Dario Jose Espinal Ruiz}

Institución Universitaria Escuela Nacional del Deporte. Valle del Cauca (Colombia) dario.espinal@endeporte.edu.co

\section{Gildardo Scarpetta Calero}

Institución Universitaria Escuela Nacional del Deporte. Valle del Cauca (Colombia)

gildardo@scarpetta@endeporte.edu.co

Natali Cruz Gonzalez

Institución Universitaria Escuela Nacional del Deporte. Valle del Cauca (Colombia)

natali.cruz@endeporte.edu.co

Para citar este artículo:

Espinal, D., Scarpetta, G. y Cruz, N. (2020). Análisis prospectivo estratégico de la educación superior en Colombia. Cultura, Educación y Sociedad, 11(1). 177-196. DOI: http://dx.doi.org/10.17981/cultedusoc.11.1.2020.13

\section{Resumen}

Las universidades deben adaptarse y anticipar los desafíos emergentes para mantener su papel en la sociedad. Este artículo tiene como objetivo proponer un marco de prospectiva educativa que facilite el análisis de los futuros alternativos en las instituciones de educación superior. Se utiliza una metodología prospectiva de tipo normativa donde se aplican entrevistas semi-estructuradas a expertos como base para un análisis morfológico que oriente la definición de escenarios futuros. Los resultados permiten caracterizar el panorama estratégico de la educación superior en Colombia y se identifican las variables de mayor influencia en los procesos de acreditación institucional. Específicamente se analiza el caso de la Institución Universitaria Escuela Nacional del Deporte IU END (Valle del Cauca, Colombia). Para esto se formulan los escenarios de futuro inercial, desarrollo incremental, cambio estructural y retroceso, los cuales conllevan a la elaboración de recomendaciones estratégicas para el escenario apuesta. Llegando a concluir que el análisis de prospectiva educativa propuesto integra aspectos institucionales, de internacionalización, ciencia, tecnología e innovación y proyección social para la construcción de escenarios alternativos de futuro útiles para la toma de decisiones estratégicas en las instituciones de educación superior.

Palabras clave: Administración educativa; universidad; cambio organizacional; innovación educativa; escenarios de futuro

\begin{abstract}
Universities must adapt and anticipate emerging challenges to maintain their role in society. This article aims to propose a framework for educational foresight that will facilitate the analysis of alternative futures in higher education institutions. A normative type of prospective methodology is used where semi-structured interviews with experts are applied as the basis for a morphological analysis to guide the definition of future scenarios. The results make it possible to characterize the strategic panorama of higher education in Colombia and identify the variables that have the greatest influence on the processes of institutional accreditation. Specifically, the case of the University Institution Escuela Nacional del Deporte IU END (Valle del Cauca, Colombia) is analyzed. For this purpose, scenarios of inertial future, incremental development, structural change and regression are formulated, which lead to the elaboration of strategic recommendations for the bet scenario. We conclude that the proposed analysis of educational foresight integrates institutional aspects, internationalization, science, technology and innovation, and social projection for the construction of alternative future scenarios useful for strategic decisionmaking in higher education institutions.

Keywords: Educational administration; university; organizational change; educational innovation; future scenarios.
\end{abstract}




\section{INTRODUCCIÓN}

Hoy, la humanidad se encuentra en el comienzo de la cuarta revolución industrial (Schwab, 2016) y el cambio hacia la economía del conocimiento es muy prometedor (Peters \& Humes, 2003). Sin embargo, los patrones de consumo, producción y empleo creados por el avance de la ciencia, la tecnología y la innovación plantean grandes desafíos que requieren una adaptación proactiva de las instituciones educativas (World Economic Forum-WEF, 2016). La rápida transformación que está viviendo la sociedad exige cambios en la educación superior; esta, por su parte, afronta numerosos y profundos desafíos para sobrevivir bajo condiciones cambiantes, lo que exige repensar drásticamente sus estrategias de desarrollo en el largo plazo (Ejdys et al., 2018).

Las universidades se han convertido en una institución relevante del mundo globalizado (Stock, 2008). Por tal motivo, deben adaptarse y anticipar los desafíos emergentes para mantener su papel en la sociedad (Labanauskis \& Ginevičius, 2017). Las innovaciones tecnológicas, las universidades con ánimo de lucro y corporativas, las nuevas modalidades de rendición de cuentas, como los rankings globales y la internacionalización, son tendencias de alto impacto en la educación superior actualmente (Barber, Donnelly \& Rizvi, 2013).

Baena (2012) y Enciso, Córdoba y Romero (2016) sostienen que las prácticas educativas contemporáneas no actualizan los conocimientos a la misma velocidad que las tendencias sociales y tecnológicas; por tanto se hace necesario un proceso de flexibilización curricular que permita la integración y participación activa de los estudiantes. El avance vertiginoso implica que los cambios en la educación no solo sean en términos del conocimiento, sino también de las instituciones y sus grupos de interés. En este sentido, la prospectiva educativa se convierte en uno de los fundamentos que permite tener una visión más amplia y profunda sobre la complejidad de las problemáticas, a las cuales se enfrentan las universidades, el sector educativo y los formuladores de políticas públicas. Al respecto Carrera, Bravo, Marín y Crasto (2013), destacan la pertinencia de la prospectiva como método de planeación que contribuye a fortalecer procesos de desarrollo social.

La prospectiva educativa es una respuesta política al surgimiento de la sociedad del conocimiento. Identifica las direcciones de transformación en la educación superior y explora la convergencia de diferentes cuerpos de conocimiento, profesiones, disciplinas y grupos de interés (Loveridge, 2009). Además, estudia la autonomía universitaria y la libertad académica, los patrones arraigados resistentes al cambio, la gobernanza organizacional y los comportamientos de actores académicos y extra-académicos (Andreescu, Gheorghiu, Zulean \& Curaj, 2012).

La prospectiva diseña múltiples caminos hacia el futuro (Gomes, 2005), lo cual facilita el encuentro de la oferta científica y tecnológica con las necesidades actuales y futuras de la sociedad (Medina y Velasco, 2014). Su naturaleza es participativa (Havas, 2005), lo que permite el aprendizaje colectivo, a partir de análisis de sistemas socio-técnicos complejos y las relaciones ciencia-sociedad (Barrè, Keenan \& Cagnin, 2006). Asimismo, la prospectiva educativa permite dilucidar el proceso para construir la reputación de una 
universidad de clase mundial (Organización de las Naciones Unidas para la Educación, la Ciencia y la Cultura-UNESCO, 2015). De manera que, la apropiación del pensamiento estratégico de largo plazo se convierte en el marco de referencia para iniciar un proceso de transformación que reconozca las capacidades necesarias para consolidarse como una universidad de clase mundial.

Una universidad de clase mundial se caracteriza por contar con aspectos institucionales consolidados, promover procesos de ciencia, tecnología e innovación, por el impacto social que genera, y tener una orientación internacional. Estos procesos son desarrollados a través de: a) una masa crítica de talento (tanto docentes como estudiantes); b) auto gobernanza y autonomía administrativa; c) libertad académica para las facultades y la investigación, que incluye el derecho al pensamiento crítico; d) el empoderamiento de los jóvenes investigadores y e) recursos suficientes para proporcionar un entorno integral para el aprendizaje y la vanguardia en la investigación y la proyección social (Salmi, 2009).

Posicionarse como una universidad de clase mundial conlleva un proyecto de innovación educativa de largo plazo (Licht, Tasiopoulou \& Wastiau, 2017). Dicho proyecto debe impulsar cambios significativos soportados en procesos, personas, tecnologías y conocimiento (Pereira y Díaz, 2018). En otras palabras, es la suma sinérgica entre crear algo nuevo, aplicar el proceso y aportar una mejora; lo cual es el resultado del proceso educativo (García, 2015). De esta manera, la universidad debe asumir una postura proactiva que le permita innovar sistemáticamente para incorporar los cambios dinámicos destinados a agregar valor al proceso educativo susceptibles de medición (Organización para la Cooperación y el Desarrollo Económicos-OCDE, 2017). Este proceso exige el uso de nuevos materiales (curriculares o tecnologías), nuevos enfoques de enseñanza (estrategias o actividades de enseñanza), el cambio de creencias (supuestos pedagógicos), la modernización de los sistemas administrativos y de los modelos de evaluación (Bryan \& Clegg, 2019).

Las nuevas formas de currículo, los nuevos métodos de enseñanza, las formas de organización y los medios educativos son temas centrales en la mayoría de debates contemporáneos sobre la innovación educativa (Whiteside, 2019). Algunas tendencias que se consolidan en la educación superior refieren a: los ecosistemas tecnológicos (Llorens, Molina, Compañ \& Satorre, 2014), la analítica académica (Baepler \& Murdoch, 2010) y del aprendizaje (Gómez-Aguilar, Hernández-García, García-Peñalvo \& Therón, 2015), la personalización del aprendizaje (Lerís, Vea y Velamazán, 2015), los Massive Open Online Courses (MOOC) (Weinhardt \& Sitzmann, 2019), la gamificación (Araya, Arias, Bottan \& Cristia, 2019), las prácticas virtuales (Blaine, 2019) y el desarrollo del pensamiento computacional (Cheung \& Abelson, 2019).

Como respuesta a este panorama, el Consejo Nacional de Educación Superior (CESU) definió los lineamientos de política pública para la educación superior que especifica el camino que debe seguir Colombia para consolidarse como uno de los países más educados de América Latina en el año 2034. En este análisis, se identificaron los problemas estructurales del sistema de educación superior colombiano, como: la falta de acceso, la permanencia, la calidad, la baja inversión en ciencia, tecnología e innovación, las asime- 
trías entre lo rural y lo urbano, el bajo nivel de internacionalización y la rigidez de los mecanismos de financiación de las universidades (CESU, 2014).

De acuerdo con este contexto, la Institución Universitaria Escuela Nacional del Deporte (Valle del Cauca, Colombia), se ha propuesto convertirse en una universidad reconocida internacionalmente por su calidad académica. Sin embargo, el avance vertiginoso de las megas tendencias globales y la problemática del sector educativo colombiano presentan un panorama incierto para las Instituciones de Educación Superior (IES). Por consiguiente, se establece, como pregunta central de investigación: ¿Cuáles son los escenarios alternativos de futuro de la Institución Universitaria Escuela Nacional del Deporte en el horizonte del 2034? Para responder este interrogante, se plantea como objetivo proponer un marco de prospectiva educativa que facilite el análisis estratégico en las instituciones de educación superior.

El artículo se organiza así: en primer lugar, se aborda el problema de investigación y se revisa la literatura de la prospectiva educativa. Enseguida, se explica la metodología prospectiva que se implementa como estrategia de investigación. Después, de manera descriptiva, se exponen los escenarios de futuro y se realizan las recomendaciones estratégicas para posicionar a la Institución Universitaria Escuela Nacional del Deporte a nivel internacional en el horizonte del 2034.

\section{MÉTodología}

Se utiliza una metodología de tipo prospectiva normativa, la cual se basa en normas, valores o estrategias que permiten dilucidar el proceso de construcción de futuro (Miklos y Tello, 2012). La unidad de análisis principal es la Institución Universitaria Escuela Nacional del Deporte, de carácter público adscrita al Ministerio del Deporte y al municipio de Santiago de Cali (Valle del Cauca, Colombia); que tiene como función desde hace 35 años la formación de talento humano en los campos de las ciencias de la educación y el deporte, la salud y la rehabilitación, y las ciencias económicas y de la administración.

El proceso investigativo se realiza en dos fases. En la fase exploratoria del estudio, teniendo como marco de análisis los factores clave para la educación superior de alta calidad que promueven el Ministerio de Educación Colombiano, los sistemas de rankings universitarios y las agencias de acreditación internacionales, se consultaron a 10 expertos por medio de una entrevista semi-estructurada que permite describir el contexto de la educación superior en Colombia e identificar las variables estratégicas con mayor influencia actual y potencial sobre la universidad colombiana. En la fase descriptiva, una vez identificadas las variables clave, se elabora un cuestionario estructurado y se consultaron a 101 actores relevantes ${ }^{1}$ a nivel nacional que pertenecen a la comunidad educativa de la Institución Universitaria Escuela Nacional del Deporte, lo que permitió elaborar los escenarios alternativos de futuro y la apuesta estratégica en el horizonte del 2034 .

\footnotetext{
1 Según Leopkey \& Parent (2016) los actores son cualquier persona u organización que tenga un interés o participación directa o indirecta en las actividades de una organización en particular, ya sea, en la gobernanza de la organización, en el dialogo, en los procesos de toma de decisiones o contribuyen al cumplimiento de los objetivos. También son denominados grupos de interés o stakeholders.
} 


\section{Participantes}

En la fase exploratoria del estudio, el criterio de selección de los expertos se realiza considerando las siguientes características: todos los participantes deben tener al menos 10 años de experiencia vinculados directamente con la educación superior en altos cargos administrativos o académicos, ya sea, a nivel universitario, en entidades reguladores del sistema de educación superior en los niveles nacionales o territoriales, o vinculados al Ministerio de Educación Nacional. Utilizando la estrategia de muestreo cualitativo por conominación ${ }^{2}$ (Flick, 2004) se seleccionan los expertos que cumplieran el perfil requerido, y de manera progresiva cada persona empezó a sugerir a otros expertos que pudieran hacer una contribución notable al estudio.

En la fase descriptiva del estudio, se seleccionan por conveniencia ${ }^{3}$ (Flick, 2004) un total de 101 actores de la comunidad universitaria de la Institución Universitaria Escuela Nacional del Deporte. El criterio de selección se realiza considerando las siguientes características: todos los participantes deben ocupar posiciones relevantes que representen los distintos grupos de interés como lo son: estudiantes, profesores, directivos, egresados y empleadores. Los participantes seleccionados se clasifican de la siguiente forma: sociedad civil organizada (egresados, empleadores; 25\%), sector privado (egresados, empleadores; $22 \%$ ), sector público (egresados, empleadores; 25\%), sector académico (estudiantes, profesores, directivos; 28\%). Entre las personas seleccionadas, el $47.3 \%$ son mujeres con una edad media de 38 años, y el 52.7\% son hombres con una edad media de 43 años.

\section{Instrumentos}

En la fase exploratoria de la investigación, se aplica una entrevista semi-estructurada, la cual mantiene su carácter abierto y permite diseñar preguntas previamente en forma de guía (Flick, 2004). Las categorías que estructuran la entrevista son: a) el contexto de la educación superior en Colombia; y las b) las variables estratégicas con mayor influencia actual y potencial sobre el sistema de educación superior colombiano. Las entrevistas se realizan de manera presencial en ambientes privados para respetar el anonimato de los expertos, su duración es aproximadamente de 45 a 60 minutos cada una.

En la fase descriptiva, como resultado del análisis de las entrevistas a expertos, se construye un cuestionario estructurado ${ }^{4}$ para la elaboración de los escenarios de futuro, organizado en cuatro dimensiones que según Salmi (2009) son consideradas como estratégicas para las universidades de clase mundial, estas son: a) aspectos institucionales; b) internacionalización; c) proyección social y d) ciencia, tecnología e innovación. El cuestionario se aplica de manera virtual respetando el consentimiento informado a través de un formulario de google a 315 personas, de las cuales se obtienen 101 respuestas validas que son incluidas en el estudio.

\footnotetext{
2 El muestreo por co-nominación consiste en pedir a los informantes que recomienden a posibles participantes. También se denomina muestreo nominado, en bola de nieve o muestreo en cadena

3 El muestreo por conveniencia es un proceso deliberado donde se seleccionan los participantes que de acuerdo a las necesidades de información que requiere el estudio.

4 Según Flick (2004) un cuestionario estructurado es aquel en el que las preguntas y posibles respuestas están formalizadas y estandarizadas.
} 


\section{Análisis de la información}

En la fase exploratoria, las entrevistas son grabadas en audio, transcritas y analizadas utilizando el software Atlas.ti aplicando el método del análisis de contenido por categorías (Bardin, 2011). Se realiza una codificación inicial basada en las dimensiones estratégicas de las universidades de clase mundial como marco general. El resultado del análisis permite identificar las categorías emergentes para la configuración de las variables estratégicas. Con el fin de controlar la concordancia interpretativa, los investigadores se encargan de la revisión paralela de la documentación y el análisis de las entrevistas de manera independiente.

En la fase descriptiva, se utiliza el método prospectivo del análisis morfológico (Godet, 2000), cuyo objetivo es guiar a los grupos de interés a construir de manera estructurada los escenarios de futuro posibles para la Institución Universitaria Escuela Nacional del Deporte en el horizonte temporal del 2034, siendo este el periodo de tiempo definido en la política pública de la educación superior colombiana. Para la construcción de los escenarios, las variables clave son evaluadas de manera descriptiva de acuerdo con la escala de escenarios posibles de Masini \& Medina (2000): escenario inercial, escenario de desarrollo incremental, escenario de cambio estructural y escenario de retroceso (Tabla 1).

TABLA 1.

Escala de evaluación prospectiva

\begin{tabular}{cc}
\hline Escenario de futuro & Descripción \\
\hline Inercial & Continuidad de las tendencias existentes \\
Desarrollo incremental & Mejoramiento de la situación actual \\
Retroceso & Deterioro de la situación actual \\
Cambio estructural & Máximo grado de transformación \\
\hline
\end{tabular}

Fuente: Masini \& Medina (2000).

Los escenarios de futuro permiten esquematizar una determinada interpretación de la realidad y describir la transición de un sistema social desde una situación presente hacia una futura. El escenario inercial se caracteriza por mostrar lo que sucederá, si los factores clave siguen su curso tendencial. Se define por los aspectos históricos, o nuevos, que inciden en el reforzamiento de las tendencias actuales. El escenario de desarrollo incremental plantea ajustes razonables que se pueden presentar en el corto o mediano plazo, partiendo de las condiciones de competitividad existentes. El escenario de cambio estructural implica una transformación profunda y estructurada, sobre procesos de ruptura que quiebran las tendencias existentes. Finalmente, el escenario de retroceso contempla el deterioro de la situación actual por causa de factores desestabilizantes, inesperados o descontrolados (Masini \& Medina, 2000). 


\section{Resultados}

\section{El contexto de la educación superior en Colombia}

Según los expertos consultados, la educación superior en Colombia se enfrenta a una serie de desafíos que condicionan su panorama de desarrollo. Las asimetrías de calidad denotan una amplia diferencia entre distintos tipos de instituciones públicas y privadas; especialmente, en las zonas rurales, donde no existe una oferta académica que atienda las necesidades de la población con programas pertinentes para estos contextos. "Las brechas entre la educación urbana y rural son escandalosas, los jóvenes rurales que quieren acceder a la educación superior tienen que desplazarse grandes distancias, ya que la internet no tiene suficiente cobertura en el campo" (Experto 3, comunicación presencial, 15/05/2019). Esto acrecienta las brechas socio- económicas entre lo rural y lo urbano. Los altos costos de la universidad privada de calidad, la desfinanciación de la universidad pública y el bajo impacto de la educación virtual y a distancia no han permitido aumentar la cobertura de la educación superior en los niveles esperados.

La falta de inversión suficiente en ciencia, tecnología e innovación ha tenido como consecuencia un bajo impacto de la universidad colombiana en el desarrollo social y productivo del país; reflejado en la poca transferencia de la innovación hacia los sectores sociales y empresariales. "Parece que la universidad va a un ritmo diferente que la industria, su capacidad de respuesta no es acorde con las necesidades del sector productivo, por tal motivo, existe un divorcio entre ambos" (Experto 7, comunicación presencial, 19/05/2019). Como consecuencia, la divergencia de intereses entre ambos sectores ha ocasionado que el sector privado no demuestre un interés permanente por financiar procesos investigativos que tengan un retorno económico y social para la industria.

Una de las tendencias con mayor influencia potencial sobre la educación superior en Colombia se orienta hacia el papel que juega la universidad en un entorno determinado por la competitividad de las ciudades-estado. "Los municipios deben encontrar una apuesta productiva que los diferencie y atraiga inversiones de manera inteligente" (Experto 9, comunicación presencial, 21/05/2019). La concentración de los recursos y capacidades en espacios territoriales reducidos permite lograr la especialización inteligente, el networking, la clusterización y la eficiencia en costos sobre la base de las ventajas competitivas y comparativas locales. Esto implica que resulta necesario definir prioridades de política pública, articular esfuerzos, asignar roles y responsabilidades, y monitorear los avances de manera sistemática para lograr construir el desarrollo sostenible en los territorios.

Tanto la integración de la universidad en los sistemas productivos locales (a través de la relación con la empresa y el estado) como el concepto de globalización exigen que la oferta académica se adapte a un contexto globalizado; pero con el propósito de atender los problemas locales. La Cuarta Revolución Industrial demanda a las instituciones educativas asumir una cultura de lo digital, para responder rápidamente a las necesidades de las comunidades, y "proponer soluciones innovadoras a los 
desafíos del desarrollo sostenible, a través de la aceleración de iniciativas spin off y start up, que emerjan del espacio universitario" (Experto 2, comunicación presencial, 08/05/2019).

En lo relacionado con la innovación educativa, "es necesario fomentar la incorporación de la formación de ciudadanos cosmopolitas e interculturales que comprendan las problemáticas globales, y que tengan la capacidad de reinventar sus profesiones permanentemente para afrontar problemáticas complejas" (Experto 5, comunicación presencial, 17/05/2019). Esto implica la alineación de habilidades duras, como: la Ciencia, la Tecnología, la Ingeniería y las Matemáticas (Science, Technology, Engineering and Mathematics-STEM), con las habilidades blandas, como: la creatividad, el trabajo en equipo, la resiliencia y el pensamiento crítico. Todo esto, mediado por la educación virtual y la inteligencia artificial, puesto que son estrategias esenciales para romper las barreras geográficas, demográficas y de desigualdad social.

Para interpretar el panorama estratégico, al cual se enfrenta el sistema de educación superior en Colombia, es indispensable conocer las brechas frente al contexto internacional y su relación con las tendencias en el marco de la innovación educativa, como elemento acelerador del cambio institucional. Existe una enorme evidencia que define las barreras que limitan el crecimiento del sistema colombiano de educación superior. No obstante, el actuar de los grupos de interés, basado en los diagnósticos locales y las tendencias globales, no se ha dado con la suficiente velocidad que demandan los procesos de transformación.

Por este motivo, es urgente que la universidad concrete su apuesta de futuro; de esta manera, podrá consolidar el cambio estructural que requiere para asumir los retos establecidos por la agenda del desarrollo sostenible y la emergencia de la Cuarta Revolución Industrial; dos paradigmas dominantes en la agenda futura de la humanidad. De acuerdo con este panorama, se ha propuesto definir los escenarios alternativos de futuro que debe prever la Institución Universitaria Escuela Nacional del Deporte para la construcción de una apuesta estratégica que le permita conocer, según la visión de sus grupos de interés, los retos de desarrollo institucional en el largo plazo.

\section{Escenarios de futuros de la IU END en el horizonte del 2034}

Para la elaboración de los escenarios de futuro, se toma como marco de referencia los factores clave para la educación superior de alta calidad que promueven el Ministerio de Educación Colombiano (MEN), los sistemas de rankings universitarios y las agencias de acreditación internacionales. Según el criterio de los expertos, se identificaron 42 variables estratégicas organizadas en las dimensiones de las universidades de clase mundial propuestas por Salmi (2009) (Tabla 2). Las variables identificadas son sometidas a la evaluación por parte de los grupos de interés de la institución de acuerdo a la escala de escenarios posibles de Masini \& Medina (2000), lo que permite elaborar la narrativa de los escenarios tendencial, incremental, cambio estructural y de retroceso para la Institución Universitaria Escuela Nacional del Deporte. 
TABla 2.

Variables de análisis prospectivo

\begin{tabular}{|c|c|c|}
\hline Dimensiones & Variables estratégicas & Definición \\
\hline & Centros de regionalización & $\begin{array}{l}\text { Descentralización de las actividades académicas y administrativas para la } \\
\text { formación de capacidades en otras regiones. }\end{array}$ \\
\hline \multirow{6}{*}{$\begin{array}{l}\text { Aspectos } \\
\text { Institucionales: } \\
\text { Son las } \\
\text { características } \\
\text { necesarias de } \\
\text { la institución } \\
\text { que facilitan } \\
\text { y promueven } \\
\text { el desarrollo } \\
\text { de las labores } \\
\text { formativas, } \\
\text { académicas, }\end{array}$} & Transformación digital & $\begin{array}{l}\text { Integración de nuevas tecnologías digitales a todos los procesos institucionales } \\
\text { que permitan la creación de valor, para la comunidad académica }\end{array}$ \\
\hline & Formación de los estudiantes & $\begin{array}{l}\text { Formación de actitudes, conocimientos, capacidades y habilidades en los } \\
\text { estudiantes que acceden a la educación superior }\end{array}$ \\
\hline & Desarrollo profesoral & Preparación integral, investigativa y pedagógica de los profesores. \\
\hline & Pruebas de estado & Evaluación externa de la calidad de la educación superior. \\
\hline & Acreditación de alta calidad & $\begin{array}{l}\text { Reconocimiento, por parte del Estado, de la calidad de las Instituciones de } \\
\text { Educación Superior y de los programas académicos. }\end{array}$ \\
\hline & $\begin{array}{l}\text { Educación a distancia y } \\
\text { virtual }\end{array}$ & $\begin{array}{l}\text { Metodología de formación flexible que privilegia la mediación de las TIC en la } \\
\text { educación no presencial, o semipresencial. }\end{array}$ \\
\hline
\end{tabular}

docentes, científicas, culturales y de extensión, de las instituciones de educación superior (Ministerio de Educación Nacional, MEN, 2019).

Diversificación de la oferta académica

Transferencias de la nación, municipio y regalías

Sostenibilidad financiera

Infraestructura física y tecnológica

Bienestar institucional

Medios educativos

Escuela internacional de verano

Visibilidad en rankings académicos

Posicionamiento Talento humano de clase internacional: mundial reconocimiento internacional obtenido, ya sea por su reputación (medida subjetiva) o por la clasificación en algún ranking especializado (medida objetiva) (Salmi, 2009).

Redes académicas

Internacionalización del currículo

Bilingüismo

Cooperación internacional

Movilidad internacional

Doble titulación y homologaciones

Posicionamiento internaciona
Oferta de nuevos programas en coherencia con la naturaleza de la institución.

Incremento de los recursos financieros de parte de entes nacionales o territoriales.

Aseguramiento de los recursos financieros estables, a largo plazo

Recursos físicos y tecnológicos para la prestación de servicios educativos de calidad.

Programas que favorezcan el desarrollo humano integral de toda la comunidad académica.

Recursos, herramientas académicas y tecnológicas fundamentales para el logro de los objetivos misionales.

Espacio para conocer las últimas tendencias y avances en diferentes campos del conocimiento, en el ámbito internacional.

Sistema de evaluación que destaca las universidades -cuya excelencia académica e impacto de sus políticas de ciencia, tecnología e innovación- han generado aportes a la humanidad, a la sociedad y al desarrollo en general.

Estrategia para atraer profesores y estudiantes internacionales de alto nivel académico.

Vinculación a comunidades académicas para compartir información y emprender proyectos de colaboración de orden internacional.

Currículo con orientación internacional en contenido y/o forma. Busca preparar estudiantes para realizarse (profesional, socialmente), en un contexto internacional y multicultural. Diseñado tanto para estudiantes nacionales como para extranjeros.

Uso habitual de dos lenguas en un mismo territorio.

Acción conjunta de países con igual, o mayor nivel de desarrollo, organismos multilaterales, organizaciones no gubernamentales y de la sociedad civil, para apoyar el desarrollo económico y social. Mediante la transferencia de tecnologías, conocimientos, experiencias o recursos.

Desplazamiento de estudiantes, docentes, investigadores o administrativos hacia otra institución, para realizar semestres académicos, cursos, pasantías, prácticas, investigaciones, cotutelas.

Cursar dos grados de la misma rama simultáneamente. Para aprovechar que, por sus estrechos vínculos, sus respectivos planes de estudio son comunes. 


\begin{tabular}{|c|c|c|}
\hline Dimensiones & Variables estratégicas & Definición \\
\hline \multirow{11}{*}{$\begin{array}{l}\text { Ciencia, } \\
\text { Tecnología e } \\
\text { Innovación: } \\
\text { Proceso } \\
\text { sistemático } \\
\text { de creación, } \\
\text { aplicación y } \\
\text { desarrollo del } \\
\text { conocimiento } \\
\text { básico y } \\
\text { avanzado, para } \\
\text { el estudio y } \\
\text { solución de } \\
\text { problemas } \\
\text { fundamentados, } \\
\text { en las ciencias } \\
\text { naturales, } \\
\text { sociales o } \\
\text { humanas } \\
\text { (UNESCO, } \\
\text { 2015). }\end{array}$} & $\begin{array}{l}\text { Alianza Universidad- } \\
\text { Empresa-Estado }\end{array}$ & $\begin{array}{l}\text { Sinergia para financiar, co financiar e implementar proyectos de investigación } \\
\text { y desarrollo que atiendan problemas concretos de la sociedad y el sector } \\
\text { productivo. }\end{array}$ \\
\hline & Inversión en I+D & $\begin{array}{l}\text { Nivel de inversión de recursos financieros en programas y proyectos de } \\
\text { investigación y desarrollo. }\end{array}$ \\
\hline & $\begin{array}{l}\text { Centros y Grupos de } \\
\text { Investigación }\end{array}$ & $\begin{array}{l}\text { Conjunto de personas que se reúnen para realizar investigación en una } \\
\text { temática dada. Por ejemplo: formulan uno o varios problemas de su interés, } \\
\text { trazan un plan estratégico de largo o mediano plazo para trabajar en él y } \\
\text { producir unos resultados de conocimiento sobre el tema cuestión. }\end{array}$ \\
\hline & Patentes & $\begin{array}{l}\text { Privilegio de explotar, exclusivamente, una invención que aporte una solución } \\
\text { técnica con aplicación industrial, por un tiempo determinado. }\end{array}$ \\
\hline & Propiedad intelectual & $\begin{array}{l}\text { Protección de la innovación creada por la mente humana. Se divide en } \\
\text { derechos de autor y propiedad industrial. }\end{array}$ \\
\hline & Innovación & $\begin{array}{l}\text { Cambio que introduce novedades o invenciones. Consiste en modificar } \\
\text { elementos con el fin de mejorarlos o renovarlos, ya sea en tecnologías, } \\
\text { servicios, procesos o aspectos sociales. }\end{array}$ \\
\hline & Proyectos Spinof y Startup & $\begin{array}{l}\text { Iniciativas empresariales promovidas desde la comunidad universitaria. Se } \\
\text { caracterizan por basar su actividad en la explotación de nuevos procesos, } \\
\text { productos o servicios, a partir del conocimiento adquirido y los resultados } \\
\text { obtenidos en la propia Universidad. }\end{array}$ \\
\hline & $\begin{array}{l}\text { Desarrollo de capacidades } \\
\text { para la CTI }\end{array}$ & $\begin{array}{l}\text { Formación de talento humano especializado, para el desarrollo de actividades } \\
\text { de ciencia, tecnología e innovación. }\end{array}$ \\
\hline & Parques tecnológicos & $\begin{array}{l}\text { Concentraciones geográficas de empresas, centros de investigación } \\
\text { y proveedores de servicios, que usan el conocimiento y la tecnología } \\
\text { intensivamente para promover el empleo y la productividad. }\end{array}$ \\
\hline & Gestión del conocimiento & $\begin{array}{l}\text { Proceso de captura, distribución y uso efectivo del conocimiento generado en } \\
\text { la universidad. }\end{array}$ \\
\hline & Centros de consultoría & $\begin{array}{l}\text { Prestación de servicios profesionales en el asesoramiento a personas, } \\
\text { empresas u organizaciones; en las áreas de experticia institucionales. }\end{array}$ \\
\hline \multirow{8}{*}{$\begin{array}{l}\text { Proyección } \\
\text { social: } \\
\text { Función } \\
\text { sustantiva que } \\
\text { tiene como } \\
\text { propósito } \\
\text { propiciar y } \\
\text { establecer } \\
\text { procesos } \\
\text { permanentes } \\
\text { de interacción } \\
\text { e integración } \\
\text { con agentes } \\
\text { y sectores } \\
\text { sociales e } \\
\text { institucionales, } \\
\text { para contribuir } \\
\text { a la solución de } \\
\text { sus problemas y } \\
\text { satisfacción de } \\
\text { sus necesidades } \\
\text { de educación } \\
\text { (MEN, 2019). }\end{array}$} & $\begin{array}{l}\text { Unidad de emprendimiento y } \\
\text { desarrollo empresarial }\end{array}$ & $\begin{array}{l}\text { Unidad para el fortalecimiento de la cultura emprendedora, la asesoría y } \\
\text { aceleración de iniciativas empresariales de la comunidad universitaria. }\end{array}$ \\
\hline & Impacto de los egresados & $\begin{array}{l}\text { Reconocimiento de los egresados, por la calidad de la formación que reciben. Y } \\
\text { por destacarse en su desempeño en la disciplina, profesión, ocupación u oficio } \\
\text { correspondiente. }\end{array}$ \\
\hline & Impacto social & $\begin{array}{l}\text { Reconocimiento por el aporte al desarrollo socio económico que la institución } \\
\text { ha realizado en la región y en el país. }\end{array}$ \\
\hline & Eventos académicos & $\begin{array}{l}\text { Actividades de extensión, cuyo propósito es la transferencia de conocimientos } \\
\text { y experiencias a la comunidad en general. }\end{array}$ \\
\hline & $\begin{array}{l}\text { Responsabilidad social } \\
\text { universitaria }\end{array}$ & $\begin{array}{l}\text { Principios, acciones y programas de la universidad, cuyo propósito es } \\
\text { contribuir a solucionar problemas sociales, económicos y ambientales; en su } \\
\text { espectro de acción. }\end{array}$ \\
\hline & Innovación social & $\begin{array}{l}\text { Nuevas ideas (productos, servicios y modelos) que satisfacen, } \\
\text { simultáneamente, necesidades sociales, y crean nuevas relaciones de } \\
\text { colaboración. }\end{array}$ \\
\hline & Desarrollo sostenible & $\begin{array}{l}\text { Políticas para satisfacer las necesidades del presente sin comprometer la } \\
\text { capacidad de las futuras generaciones. Que garantizan el equilibrio entre el } \\
\text { crecimiento económico, el cuidado del medio ambiente y el bienestar social. }\end{array}$ \\
\hline & $\begin{array}{l}\text { Conservación y protección del } \\
\text { medio ambiente }\end{array}$ & $\begin{array}{l}\text { Principios y acciones para regular, minimizar o impedir el daño que las } \\
\text { actividades de índole industrial, agrícola, urbana, comercial (o de otro tipo) } \\
\text { ocasionan a los ecosistemas naturales. }\end{array}$ \\
\hline
\end{tabular}

Fuente: Elaboración propia a partir de Salmi (2009), UNESCO (2015), MEN, 2019. 
En el escenario de cambio estructural resalta la dimensión de aspectos institucionales (Figura 1). Las variables estratégicas que sobresalen para tener en cuenta en un proceso de cambio institucional son: la transformación digital de los procesos académicos y administrativos, la sostenibilidad financiera, la diversificación de la oferta académica y el mejoramiento de los resultados en las pruebas de estado. Mientras que, en un escenario de mejoramiento incremental, se destacan factores como: el bienestar de estudiantes y trabajadores, la educación a distancia y virtual, la acreditación en alta calidad y la formación de estudiantes.

El resultado anterior implica que la Institución Universitaria Escuela Nacional del Deporte, tendrá que realizar un proceso de transición en el mediano plazo para lograr el cambio estructural. Partiendo del mejoramiento incremental que se fije en aspectos institucionales relacionados con el funcionamiento interno hasta llegar al cambio estructural que asegure su competitividad en un contexto globalizado. Es necesario tener en cuenta que el factor de profesores sobresale en un escenario de retroceso. Esto indica que la apuesta institucional por el desarrollo profesoral se convierte en un elemento impulsor, o retardador, del proceso de cambio. Por ello, la transición hacia un escenario de cambio estructural dependerá, en gran medida, de la mediación de los profesores.

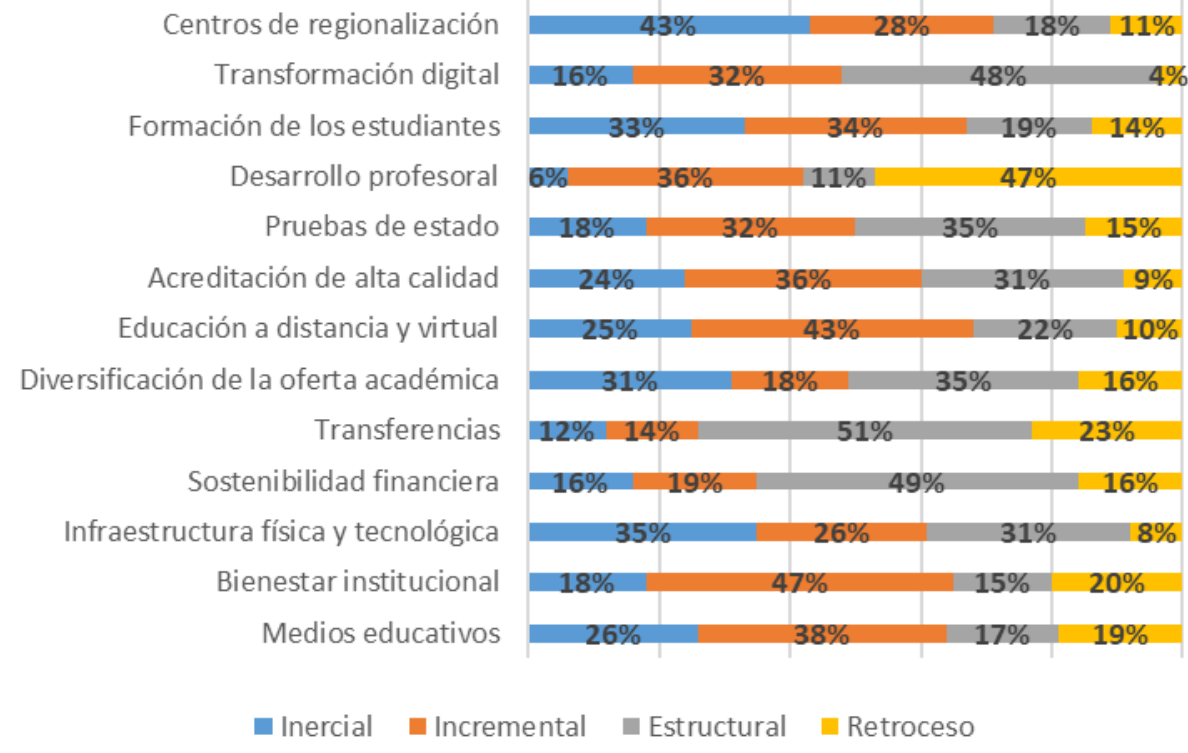

Figura 1. Subsistema aspectos institucionales Fuente: Elaboración propia.

En el subsistema de posicionamiento internacional (Figura 2), se destaca el escenario de cambio estructural. Las variables internacionalización del currículo, talento humano de clase mundial y visibilidad en rankings internacionales, se convierten en aspectos estratégicos para tener en cuenta en el proceso de conversión de la institución en un centro de excelencia académica de nivel internacional. En un escenario de cambio incremental, resulta necesario impulsar las dobles titulaciones y homologaciones con universidades del extranjero. Como también, consolidar los procesos de cooperación internacional y bilingüismo, para garantizar la formación de capacidades que permitan preparar la institución en sus procesos de internacionalización. 


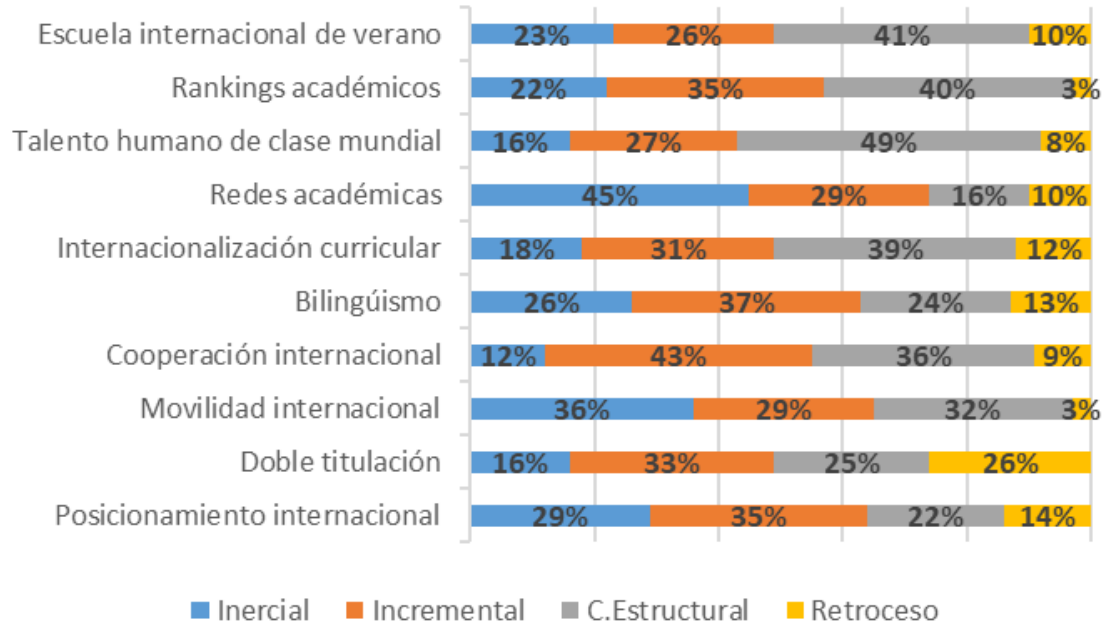

Figura 2. Sub sistema posicionamiento internacional Fuente: Los autores.

En el subsistema de ciencia, tecnología e innovación (Figura 3), prevalece el escenario del cambio estructural. Las variables asociadas a la generación, transferencia, aplicación y protección del conocimiento son las que definen los procesos de transformación, para consolidarse internacionalmente. Se destacan aspectos como: la gestión del conocimiento, la creación de un parque tecnológico, la aceleración de compañías basadas en el modelo spin off y start up. Todo esto, para incrementar el impacto de la innovación, la gestión de la propiedad intelectual, especialmente de las patentes que permitan proteger y explotar las invenciones de los proyectos de investigación.

No obstante, resulta crucial incrementar las fuentes de financiación, a través de la puesta en marcha del Comité Estado-Empresa-Universidad. Asimismo, se debe definir una agenda estratégica de ciencia, tecnología e innovación acorde con las necesidades y problemáticas de los distintos sectores sociales y productivos. Puesto que, al verse en riesgo la financiación de este subsistema, emerge el escenario de retroceso institucional, el cual condiciona, en gran medida, el proceso de cambio.

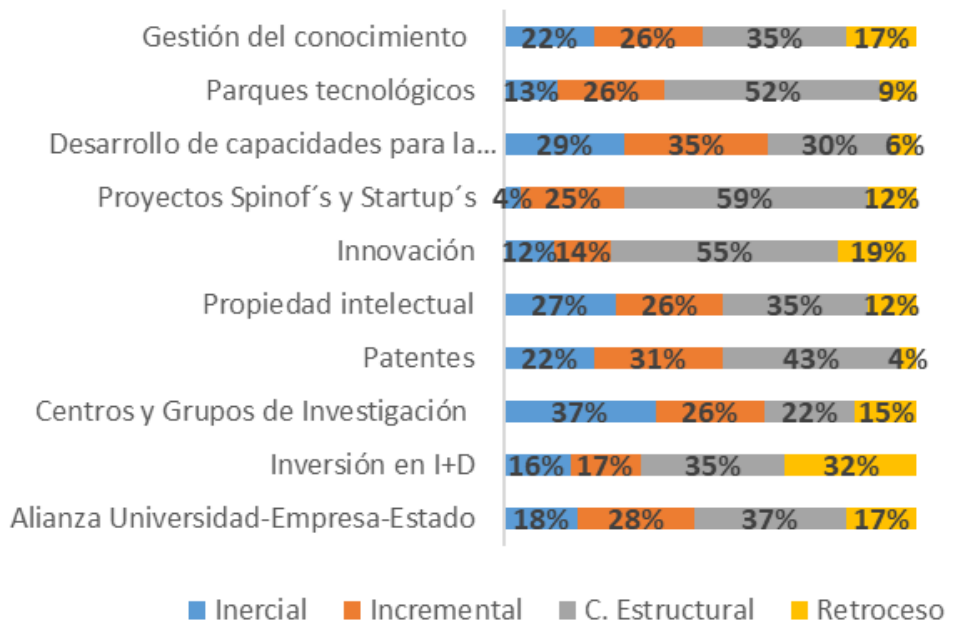

Figura 3. Sub sistema ciencia, tecnología e innovación Fuente: Elaboración propia. 
En el subsistema de proyección social (Figura 4), prevalece el escenario del cambio estructural. El aumento del impacto social de la institución se convierte en factor estratégico; esto se logra por medio de la innovación social y la responsabilidad social universitaria, que son variables determinantes. También, emergen, como unidades estratégicas, la puesta en marcha de centros de consultoría. Con el fin de que se aprovechen las capacidades institucionales y la consolidación de las unidades de emprendimiento y desarrollo empresarial. Así, se potencia la cultura emprendedora y la generación de empleo para los estudiantes.

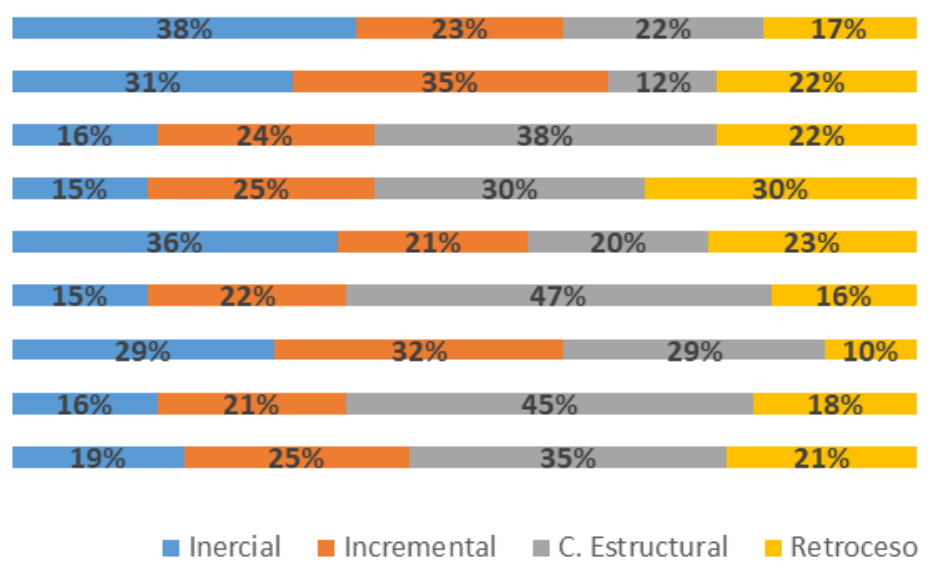

Figura 4. Subsistema proyección social Fuente: Elaboración propia.

Un escenario es una herramienta de análisis político que describe un conjunto posible de condiciones de futuro (Schwartz, 1991), en un contexto que se caracteriza por ser complejo e incierto (Glenn \& Gordon, 2004; Coates, 2010). Por tanto, es un instrumento para disminuir el nivel de incertidumbre y el margen de error en la toma de decisiones (Medina, Becerra y Castaño, 2014; Schwartz y Medina, 1995; Masini \& Medina, 2000). En este sentido, la Institución Universitaria Escuela Nacional del Deporte en su proceso de cambio institucional puede utilizar los escenarios alternativos de futuro identificados para probar o reformular sus estrategias plasmadas en su plan de desarrollo, escanear o explorar los cambios en el contexto de la educación superior y evaluar la progresión en su proceso de transformación como una universidad de nivel internacional reconocida por su calidad académica.

\section{DisCUSIÓN}

El proceso de transición de la Institución Universitaria Escuela Nacional del Deporte, tanto en el ámbito local como internacional, implica consolidar un marco que ayude a delinear el proceso de cambio organizacional. Para esto, se propuso un modelo de prospectiva educativa. En el que se integren aspectos institucionales involucrados en los procesos académicos y administrativos.

Los referidos procesos, a su vez, brindan soporte a la dinámica interna de la universidad. En otras palabras, debe existir: a) una estructura de internacionalización, cuyo propósito 
sea consolidar una gobernanza fundamentada en la integración con la comunidad académica global; b) un sistema universitario de ciencia, tecnología e innovación, que defina la agenda estratégica de desarrollo institucional, basada en la generación, transferencia, aplicación, protección y monetización del conocimiento; y c) un programa de desarrollo social y productivo que incremente el impacto social de la institución.

El modelo de prospectiva educativa sugerido se fundamenta en el supuesto de que la estructura de gobernanza universitaria articule con el contexto internacional, en el aspecto de las necesidades locales. Se debe hacer por medio de un proyecto de innovación educativa, cuya finalidad sea acelerar la reducción de brechas frente a las universidades de clase mundial. Conocer las implicaciones de las macro tendencias globales y su impacto en la universidad permite definir el curso de acción para la emergencia de los escenarios alternativos de futuro, lo cual reduce los niveles de incertidumbre en la toma de decisiones estratégicas.

Carecer de visión prospectiva es una amenaza para las instituciones que pretenden afrontar procesos de cambio. En efecto, desconocer las señales del entorno y asumir una actitud reactiva frente al contexto globalizado de la educación superior podría desencadenar un escenario sorpresa, o catastrófico, que, si se hubiera anticipado se habría podido evitar, o al menos mitigar.

Los escenarios de futuro plantean un proceso de transición de una situación inercial hacia una de cambio estructural. Para ello, hay que desarrollar una serie de capacidades institucionales que ayuden a afrontar el reto de ser una universidad de clase mundial. Es cierto que, cuando se determinan los factores críticos que se deben tener en cuenta para emprender este proceso, estos pueden cambiar de manera vertiginosa. No obstante, es preciso definir un escenario apuesta y una visión de futuro para focalizar los esfuerzos y mantener alineados los recursos y las capacidades requeridas para consolidar la apuesta de cambio. Esta iniciativa también tiene implícita la transformación de la cultura organizacional y la consolidación de una ruta de madurez institucional que permita ir logrando, de manera paulatina, los hitos definidos en su visión de futuro.

\section{Conclusiones}

Como conclusiones de este estudio, se presentan los escenarios alternativos de futuro identificados para la Institución Universitaria Escuela Nacional del Deporte en el horizonte del 2034.

\section{Escenario inercial 2034}

La IU END, en el 2034, mantiene sus fuentes de financiación provenientes de la transferencia de recursos, por parte del Ministerio del Deporte y de la venta de servicios académicos. Su crecimiento se limita a la ampliación de su infraestructura física y tecnológica y a la implementación de centros de regionalización en el suroccidente colombiano. En esta región, se ofrecen sus programas académicos acreditados en alta calidad como: profesional en deporte, fisioterapia y administración de empresas. Sus resultados académicos se mantienen dentro del promedio nacional, como lo reflejan las pruebas de estado de 
evaluación de la calidad de la educación superior. La estrategia de internacionalización se evidencia de tres formas: a) el fomento de la movilidad de estudiantes y profesores a universidades del exterior, b) la invitación a expertos internacionales para el desarrollo de cursos y seminarios y c) la participación en redes académicas internacionales.

Para el desarrollo de sus procesos de ciencia, tecnología e innovación, la institución continúa con su apuesta hacia el fortalecimiento de los grupos de investigación y la creación del centro de investigación en ciencias del deporte y la salud. Esto implica la formación de capacidades para los investigadores que pertenecen a sus distintas facultades. Con el propósito de ampliar sus procesos de proyección social, la IU END sigue incentivando la organización de eventos académicos de alcance nacional. Sus egresados se destacan, especialmente, en el contexto del sector deporte. Y genera proyectos de sensibilización para la protección y conservación del medio ambiente, dirigidos a su comunidad académica.

\section{Escenario de desarrollo incremental 2034}

La IU END, en el 2034, logra la acreditación institucional en alta calidad. Esto se ve reflejado en los procesos de formación de estudiantes y los resultados en las pruebas de estado. Este resultado ubica a la institución dentro de las 10 mejores del país, en cada una de sus áreas de formación. Se mejora el bienestar institucional, especialmente, el de los profesores, quienes gozan de planes de incentivos que promueven el aumento de la producción intelectual. Cuenta con una de las mejores bibliotecas en ciencias de la salud y el deporte, laboratorios con tecnología de punta y salas de simulación empresarial y de negocios. Inicia una apuesta fuerte por la educación a distancia y virtual; lo que permite la presencia de la institución en todo el territorio nacional con una oferta de pregrados y posgrados lo suficientemente diversificada, para atender las necesidades de distintas regiones del país.

Su estrategia de internacionalización consiste en una oferta académica, basada en procesos de doble titulación y homologaciones, con universidades del extranjero. Lo anterior implica la incorporación de la formación bilingüe en todos sus programas académicos; así, se mejora el posicionamiento internacional de la institución; o sea, que se hace más atractiva para desarrollar proyectos de cooperación internacional con universidades del exterior. Todo esto involucra la formación de capacidades especializadas para gestionar procesos de ciencia, tecnología e innovación de alcance internacional. En efecto, articula su estrategia de crecimiento institucional con los objetivos del desarrollo sostenible y con proyectos de innovación social que impactan algunas comunidades del suroccidente colombiano.

\section{Escenario de cambio estructural 2034}

La IU END, en el 2034, logra la autosuficiencia financiera. Las transferencias, recibidas de parte de los organismos estatales, crecen constantemente y son aprovechadas como parte del presupuesto de inversión. Una de sus principales apuestas es la transformación digital y la automatización de sus procesos académicos y administrativos; lo que aumenta, de manera exponencial, sus niveles de eficiencia. Los resultados de las pruebas de estado 
la posicionan como una de las cinco mejores universidades del país en sus áreas de formación especializadas. Y su oferta académica se diversifica hacia las maestrías y doctorados, lo cual le permite obtener acreditaciones en alta calidad de alcance internacional.

La internacionalización de su currículo le permite atraer estudiantes y profesores de clase de mundial. La escuela de verano convierte a la IU END en un centro de formación académica intercultural de alto nivel científico, lo que aumenta su presencia en toda Latinoamérica. La automatización de la gestión del conocimiento permite la generación, difusión, aplicación y apropiación del conocimiento generado en los procesos de investigación. El parque tecnológico, sustentado sobre un modelo universidad-estado-empresa- se establece como un epicentro de la innovación. La generación de propiedad intelectual, especialmente de patentes, produce un flujo de recursos de inversión en ciencia, tecnología e innovación para la financiación de spinoff y startup, creada por estudiantes y profesores. La unidad de emprendimiento y desarrollo empresarial y los centros de consultoría se convierten en unidades de negocio autosuficientes que contribuyen a potenciar el impacto social de la institución, por medio de la aceleración de iniciativas que busquen la transformación social y productiva de la región y del país.

\section{Escenario de retroceso 2034}

La IU END, en el 2034, no alcanza viabilidad financiera. Sus ingresos dependen exclusivamente de la transferencia de recursos desde el Ministerio del Deporte. No puede diversificar su oferta académica en cuanto a programas, servicios de investigación, proyectos de extensión. Esto limita su presencia en la ciudad de Cali (Colombia). Como está por debajo de las 50 mejores universidades del país, no consigue las acreditaciones en alta calidad de parte del Ministerio de Educación Nacional, lo cual se refleja en los resultados de las pruebas de estado, en los últimos años. Las causas de lo anterior son: a) no cuenta con una planta de profesores calificados para este propósito, b) carece de medios educativos y c) prevalece el deterioro de la infraestructura física y tecnológica. Todo esto, debido a la falta de inversión en los aspectos institucionales y a una administración sin transparencia en el manejo de los recursos.

La institución no hace presencia internacionalmente. Ni ha logrado consolidar una política de movilidad de estudiantes y profesores que le permita participar en redes académicas y de proyectos de cooperación internacional. Debido a la crisis financiera, las inversiones en ciencia, tecnología e innovación han venido disminuyendo progresivamente. En consecuencia, los estudios de investigación se limitan a los procesos en el aula de clase y a trabajos de grado. La proyección social institucional se ve restringida a la organización de eventos académicos y algunos proyectos de responsabilidad social que le permiten cumplir con los requisitos exigidos por los organismos de control para la educación superior.

\section{Visión estratégica 2034}

El planteamiento y la definición de los distintos escenarios permiten construir un proceso de reflexión sistemática, acerca de las alternativas de futuro que enfrenta la IU END, 
en el contexto de la educación superior colombiana. Hay que recordar que dicho contexto se caracteriza por ser incierto porque está sometido a las diferentes ideologías políticas que establecen sus lineamientos de desarrollo. No obstante, cabe resaltar que los grupos de interés de la institución han seleccionado, de manera consensuada, el escenario del cambio estructural, como el que se ajusta a la visión de futuro y a los valores compartidos que caracterizan a la institución. En este sentido, se identificaron los proyectos que se deben priorizar para poder consolidar este escenario, los cuales fueron seleccionados por sus grupos de interés. La visión de futuro se construyó sobre los ejes estratégicos que delimitan el curso de acción para llegar al cambio estructural, esta se define como: "Ser una institución universitaria de alta calidad de nivel internacional que fundamenta sus procesos en un sistema de ciencia, tecnología e innovación con alto impacto social”.

\section{Limitaciones e investigación futura}

Este análisis se limita al Estudio de Caso de la Institución Universitaria Escuela Nacional del Deporte, Valle del Cauca, Colombia. Por tanto, los resultados describen la visión de sus actores, en relación con el problema de investigación planteado en el contexto de la educación superior en Colombia.

La investigación futura para este tipo de estudios, debe orientarse hacia: a) la identificación de los factores que configuren la madurez de las instituciones de educación superior en el desarrollo de programas de innovación educativa de largo plazo; b) el estudio de la influencia de los actores de la comunidad universitaria sobre el proceso de cambio institucional de largo plazo; c) el análisis de los procesos de toma de decisiones ante escenarios de alta incertidumbre y d) la gestión de la ciencia, de la tecnología y de la innovación, los contenidos curriculares y la proyección social, en el marco de los procesos de planeación de largo plazo.

\section{REFERENCIAS}

Andreescu, L., Gheorghiu, R., Zulean, M. \& Curaj, A. (2012). Systemic foresight for romanian higher education. In, A. Curaj, P. Scott, L. Vlasceanu \& L. Wilson, European higher education at the crossroads: Between the Bologna process and national reforms (pp. 995-1017). London: Springer.

Araya, R., Arias, E., Bottan, N. \& Cristia, J. (2019). Does Gamification in Education Work? Experimental Evidence from Chile. Washington: BID.

Baena, G. (Noviembre, 2012). Prospectiva de la Educación Superior. Propuestas para el futuro que ya empezó. Prospecta america latina, III Congreso Latinoamericano de prospectiva y estudios del futuro, Mendoza, Argentina.

Baepler, P. \& Murdoch, C. (2010). Academic Analytics and Data Mining in Higher Education. International Journal for the Scholarship of Teaching and Learning, 4(2), 1-9. Disponible en https://doi.org/10.20429/ijsotl.2010.040217

Barber, M., Donnelly, K. \& Rizvi, S. (2013). An avalanche is coming: higher education and the revolution ahead. London: IPPR.

Bardin, L. (2011). Analisis de contenido. Sao Paulo: Ediciones 70. 
Barrè, R. Keenan, M. \& Cagnin, C. (2008). Future-Oriented Technology Analysis: Future Directions.En, C. Cagnin, M. Keenan, R. Johnston, F. Scapolo \& R. Barrè, Future-Oriented Technology Analysis (163-169). Berlin: Springer. https://doi. org/10.1007/978-3-540-68811-2

Blaine, A. (2019). Interaction and presence in the virtual classroom: An analysis of the perceptions of students and teachers in online and blended Advanced Placement courses. Computers \& Education, 132(1), 31-43. https://doi.org/10.1016/j.compedu.2019.01.004

Bryan, C. \& Clegg, K. (2019). Innovative assesment in higher education. New York: Routledge.

Carrera, M., Bravo, O., Marín, F. y Crasto, C. (2013). Futuribles y futurables universitarios para el desarrollo sostenible del estado Falcón. Revista Lider, 22(15), 39-74. Disponible en http://ceder.ulagos.cl/lider/images/numeros/22/2.LIDER\%2022_Carrera_pp39_74.pdf

Cheung, S. \& Abelson, H. (2019). Computational Thinking Education. Singapore: Springer.

Coates, J. (2010). The future of foresight-A US perspective. Technological Forecasting \& Social Change, 77(9), 1428-1437. https://doi.org/10.1016/j.techfore.2010.07.009

Ejdys, J., Gudanowska, A., Halicka, K., Kononiuk, A., Magruk, A., Nazarko, J., Nazarko, L., Szpilko, D. \& Widelska, U. (2018). Foresight in higher education institutions: Evidence from Poland. Foresight and STI governance, 13(1), 77-89. https:// doi.org/10.17323/2500-2597.2019.1.77.89

Enciso, J. E., Córdoba, L. y Romero, L. C. (2016). Adaptaciones curriculares para el ingreso, permanencia y graduación de estudiantes con discapacidad: una experiencia desde la educación superior. Cultura Educación y Sociedad, 7(2), 72-93. Recuperado de https://revistascientificas.cuc.edu.co/culturaeducacionysociedad/article/ view/1103

Flick, U. (2004). Introducción a la investigación cualitativa. Madrid: Morata.

García, F. (2015). Mapa de tendencias en innovación educativa. Education in the Knowledge Society Journal, 16(4), 6-23. Disponible en https://revistas.usal.es/index.php/eks/article/view/13493/0

Glenn, J. y Gordon, T. (2004). Metodología de investigación de futuros. metodología prospectiva; the millennium project. Buenos Aires: American Council for the United Nations University.

Godet, M. (2000). La Caja de Herramientas de la Prospectiva Estratégica. París: LIPSOR-CNAM.

Gomes, A. (2005). Proyecto Quo Vadis: El futuro de la investigación agrícola y la innovación institucional en América Latina y el Caribe. Quito: Red Nuevo Paradigma.

Gómez-Aguilar, D., Hernández-García, Á., García-Peñalvo, F. \& Therón, R. (2015). Tap into visual analysis of customization of grouping of activities in eLearning. Computers in Human Behavior, 47, 60-67. https://doi.org/10.1016/j.chb.2014.11.001

Havas, A. (2005). Terminology and methodology for benchmarking foresight programmes. [Proyecto ForSociety]. 
Labanauskis, R. \& Ginevičius, R. (2017). Role of stakeholders leading to development of higher education services. Engineering Management in Production and Services, 9(3), 63-75. https://doi.org/10.1515/emj-2017-0026

Leopkey, B. \& Parent, M. (2016). Stakeholder perspectives regarding the governance of legacy at the olympic games. Annals of Leisure Research, 18(4), 528-548. https:// doi.org/10.1080/11745398.2015.1092388

Lerís, D., Vea, F. y Velamazán, Á. (2015). Aprendizaje adaptativo en Moodle: Tres casos prácticos. Education in the Knowledge Society, 16(4), 138-157. https://doi. org/10.14201/eks201516138157

Licht, A., Tasiopoulou, E. \& Wastiau, P. (2017). Open Book for Educational Innovation. Brussels: European Schoolnet.

Llorens, F., Molina, R., Compañ, P. \& Satorre, R. (2014). Technological Ecosystem for Open Education. In, R. Neves-Silva, G. Tshirintzis, V. Uskov, R. Howlett, L. Jain, Smart Digital Futures 2014, 262, (pp. 706-715). https://doi.org/10.3233/978-161499-405-3-706

Loveridge, D. (2009). Foresight: The art and science of anticipating the future. New York: Routledge.

Masini, E. \& Medina, J. (2000). Scenarios as seen from a human and social perspective. Technological Forecasting and Social Change, 1(65), 49-66. https://doi. org/10.1016/S0040-1625(99)00127-4

Medina, J. y Velasco, C. (2014). Desafios para la transformacion productiva y educativa: hacia una sociedad y economia del conocimiento. Cali: Universidad Autonoma de Occidente.

Medina, J., Becerra, S. y Castaño, P. (2014). Prospectiva y política para el cambio estructural en América Latina y el Caribe. Santiago de Chile: CEPAL.

Miklos, T. y Tello, M. (2012). Planeacion prospectiva: una estrategia para el diseño del futuro. México, D.F.: Fundacion Javier Barros Sierra.

OCDE. (2017). The OECD Handbook for Innovative Learning Environments. París: OECD.

Pereira, A. y Díaz, D. (2018). Inclusión digital y los retos para la innovación educativa en Brasil y Colombia. Revista Iberoamericana de Psicología, 11(3), 82-88. https:// doi.org/10.33881/2027-1786.rip.11307

Peters, M. \& Humes, W. (2003). Education in the Knowledge Economy. Policy Futures in Education, 1(1), 1-19. https://doi.org/10.2304/pfie.2003.1.1.1

República de Colombia. CESU. (2014). Propuesta de política pública para la excelencia de la educación superior en Colombia en el escenario de la paz. [Acuerdo por lo superior 2034]. Bogotá D.C.: Consejo Nacional de Educación Superior. Disponible en https://www.mineducacion.gov.co/1621/w3-article-344500.html

República de Colombia. MEN. (25 de julio de 2019). Por el cual se sustituye el Capítulo 2 y se suprime el Capítulo 7 del Título 3 de la Parte 5 del Libro 2 del Decreto 1075 de 2015 -Único Reglamentario del Sector Educación. [Decreto 1330]. Diario Oficial: 51.025 .

Salmi, J. (2009). El desafio de crear universidades de clase mundial. Washington: Banco Mundial. 
Schwab, K. (2016). La cuarta revolución industrial. Barcelona: Peguin Random House. Schwartz, P. (1991). The Art of the Long View: Planning for the Future in an Uncertain World. New York: Doubleday.

Schwartz, P. y Medina, J. (1995). La planificación estratégica por escenarios. Cuadernos de Administración, 14(21), 199-225. https://doi.org/10.25100/cdea.v14i21.114

Stock, G. (2008). The current status and the future of universities within society. In, L. Engwall \& D. Weaire, The University in the Market (pp. 77-89). London: Portland Press.

UNESCO. (2015). Unesco science report: Towards 2030. París: Unesco.

Weinhardt, J. \& Sitzmann, T. (2019). Revolutionizing training and education? Three questions regarding massive open online courses (MOOCs). Human Resource Management Review, 29(2), 218-225. https://doi.org/10.1016/j.hrmr.2018.06.004

Whiteside, T. (2019). The sociology of educational innovation. New York: Routledge. WEF. (2016). New Vision for Education. Davos: World Economic Forum. 